\title{
ECUADOR: EL NUEVO SISTEMA POLÍTICO EN FUNCIONAMIENTO
}

\author{
Ecuador: New Political System Into Operation
}

\section{SIMÓN PACHANO}

Facultad Latinoamericana de Ciencias Sociales, FLACSO, Ecuador

\begin{abstract}
RESUMEN
La vigencia de la nueva Constitución y la elección de nuevas autoridades debían llevar al cierre definitivo de una larga etapa de inestabilidad política en Ecuador. El fuerte apoyo obtenido por el presidente Correa a lo largo de su gestión y su reelección en primera vuelta podían interpretarse como signos positivos para alcanzar esos objetivos. Sin embargo, tres aspectos aparecen como obstáculos para la instauración plena de un nuevo orden que garantice la gobernabilidad y la estabilidad en un contexto de avances en la representación y la participación. Primero, la permanencia de los aspectos más negativos del diseño institucional, que no fueron parte de la reforma constitucional. Segundo, el liderazgo personal y hegemónico del Presidente que actúa como obstáculo para la institucionalización del proceso. Tercero, la concepción de éste como una revolución, que incentiva la polarización y alimenta el juego de ganadores y perdedores absolutos.
\end{abstract}

Palabras clave: Ecuador, Constitución, Presidente Correa, elecciones.

\begin{abstract}
The validity of the new Constitution and election of new state authorities should lead to the definitive closure of a long period of political instability in Ecuador. The strong support received by President Correa during his administration and his reelection in the first round could be interpreted as positive signs for achieving those goals. However, three aspects appear to be obstacles to the fully establishment of a new order able to ensure good governance and stability, in a context where progress has been made as to popular representation and participation. First, the permanence of negative aspects of institutional design that were not made part of the constitutional reform. Second, hegemonic leadership by the President which serves as an obstacle to the institutionalization of the process. Third, the notion of the process as a revolution, which encourages polarization and feeds a game of absolute winners and losers.
\end{abstract}

Key words: Ecuador, Constitution, President Correa, elections. 


\section{CRONOLOGÍA DE LOS EVENTOS MÁS IMPORTANTES DE 2009}

\section{Coyuntura política y social}

En el año 2009 debía concluir el proceso de reforma política iniciado dos años antes. En su discurso de posesión, en enero de 2007, el presidente Rafael Correa reiteró su decisión de convocar a una Asamblea Constituyente para realizar una profunda reforma del sistema político ecuatoriano. En noviembre de 2008 se aprobó, mediante referéndum, la Constitución elaborada por la Asamblea Constituyente que previamente fue convocada por medio de otro referéndum. La conclusión de ese proceso debía materializarse en tres hitos: a) vigencia plena de la nueva Constitución; b) realización de las elecciones presidenciales, legislativas y de organismos subnacionales; c) expedición de un conjunto de leyes que daría forma final al diseño institucional. ${ }^{1}$ Con ello se buscaba poner fin al ciclo de inestabilidad que afectó a Ecuador desde mediados de la década de los noventa. ${ }^{2}$

Con la Constitución ya en vigencia, en abril de 2009 se realizaron las elecciones generales para elegir presidente y vicepresidente de la República, 124 asambleístas (legisladores), 23 prefectos y viceprefectos provinciales, 221 alcaldes municipales, 1.039 concejales urbanos, 542 concejales rurales y 5 diputados para el Parlamento Andino. Por tanto, se renovaban todos los cargos de elección popular y se dio inicio al que, según las disposiciones acerca de la reelección, sería considerado como el primer período de gestión de cada una de las autoridades. ${ }^{3}$ Como se verá en la segunda sección, los resultados de esta elección mantuvieron y profundizaron la tendencia de transformación integral del sistema de partidos que venía manifestándose por lo menos desde el año 2002. Asimismo, en ésta se rompió la tradición imperante desde 1979 de la necesidad de ir a una segunda vuelta para definir la elección presidencial.

Las leyes que debían aprobarse están definidas por la Constitución (en su primera disposición transitoria), de manera que constituían un mandato obligatorio para la Asamblea Nacional. Entre estas se incluían las que regirían a algunos organismos del sector público (Corte Constitucional, Defensoría Pública), además de las que se orientaban a la ejecución de políticas públicas (como las de regulación de los recursos hídricos, educación, cultura,

1 Después de un período de enfrentamiento con el Congreso Nacional, el Presidente convocó a un referéndum, en abril de 2007, para consultar sobre la instalación de la Asamblea Constituyente. Esta propuesta tuvo el $82 \%$ de aprobación, a pesar de que para llegar a la convocatoria el gobierno recurrió (por medio del Tribunal Supremo Electoral) a la destitución inconstitucional de 57 diputados. En septiembre de 2007 se realizaron las elecciones para asambleístas constituyentes, en las que el Movimiento Alianza País (AP), constituido para la campaña de Rafael Correa del año 2006, obtuvo el 56,2\% de los escaños. De esta manera, el movimiento gubernamental pudo mantener el control total de la Asamblea.

2 El objetivo que guió a todo el proceso era la refundación del país, aunque no se lo planteó en esos términos por la previa utilización de ese calificativo por el gobierno de Alfredo Palacio (2005-2007). La propuesta incluía profundas reformas en lo político, en lo económico y en lo administrativo. Estaba orientada a mejorar la gobernabilidad y a establecer un nuevo modelo económico que pusiera fin a la "larga y oscura noche neoliberal" (entrevista al presidente Correa, diario Hoy: domingo 10 de junio de 2007).

3 La nueva Constitución establece el límite de una sola reelección (consecutiva o no) para todos los cargos de elección popular (CP, artículo 114). Sin embargo, al considerar a ésta como la primera elección, quienes se reeligieron (entre ellos el presidente de la República y varios alcaldes y prefectos) podrán optar por la reelección al finalizar este período. 
deporte, registro de datos, registro civil), así como otras que estaban dirigidas a dar forma a las nuevas instituciones y procedimientos que se introdujeron con el nuevo marco jurídico (como las de comunicación, descentralización territorial, participación ciudadana).

Con la aprobación de las leyes y la elección de nuevas autoridades debía iniciarse la segunda fase de la revolución ciudadana propuesta por Rafael Correa desde la campaña electoral e iniciada en su primer mandato (2007-2009). Esta nueva fase debía orientarse básicamente hacia la institucionalización del proceso, en el que la nueva Constitución se convertiría en el elemento sobre el cual descansaría un nuevo orden político, económico, social y cultural. ${ }^{4}$ Pero, en sentido contrario a estos objetivos, y más bien siguiendo las pautas tradicionales de la política ecuatoriana de los quince años previos, el día que entró en vigencia fue objeto de la primera violación cuando el Tribunal Constitucional -nombrado por la Asamblea Constituyente dentro de lo establecido por la anterior Constitución- se arrogó las funciones que en la nueva Carta política corresponden a la Corte Constitucional. Ese paso inicial fue seguido posteriormente por otros similares, tanto en el procesamiento de leyes como en las acciones desplegadas desde el Ejecutivo. Se cumplía así la observación de varios académicos acerca de la permanencia de las viejas prácticas políticas dentro de los nuevos moldes institucionales (Basabe, 2009; Mejía y Polga, 2010).

El factor fundamental para la instauración del nuevo ordenamiento era el apoyo masivo de la población a la gestión gubernamental. Las cifras favorables obtenidas en las encuestas fueron ratificadas en la reelección del presidente en la primera vuelta, lo que le otorgó una legitimidad de origen que se constituyó en su principal soporte. ${ }^{5}$ Sin embargo, no ocurrió lo mismo en la elección de legisladores para la Asamblea Nacional, en la que Alianza País perdió la mayoría absoluta que había tenido en la Asamblea Constituyente y, adicionalmente, reaparecieron indicios de la fragmentación que fue característica en la representación política ecuatoriana desde el inicio del período democrático. Pero, a diferencia de lo que sucedió bajo gobiernos anteriores, en esta ocasión no se estructuró una oposición que lograra capitalizar esos resultados. La casi total desaparición de los partidos políticos, el reflujo de los movimientos sociales, la ausencia de actores políticos con capacidad para formular propuestas alternativas y la desarticulación del espacio nacional como la arena política central (una de cuyas expresiones es la presencia de un alto número de movimientos provinciales entre las bancadas legislativas) fueron factores que contribuyeron a moderar el efecto del descenso relativo del gobierno. ${ }^{6}$

Desde su primera campaña, Rafael Correa estableció como objetivo central la realización de una revolución ciudadana, entendida -según el discurso de posesión de su segundo mandato- como un proceso de transformación en todos los niveles, incluyendo una transformación de las pautas culturales y de los principios éticos.

5 La media de aprobación de la gestión gubernamental fue de 57,2\% en el año 2009, el tercero del mandato de Rafael Correa (Serviquanti-Habitus, 2009). Aunque esto significó una leve baja en relación al 60,9\% del año anterior, constituye una cifra muy alta en la historia reciente de Ecuador. Los anteriores gobernantes mantuvieron una aprobación significativamente inferior, como lo ilustra la aprobación de 20,8\% que obtuvo el anterior presidente a la misma altura de su mandato (Serviquanti-Habitus, 2004). En la elección presidencial Rafael Correa obtuvo el 52,0\%, a 24 puntos porcentuales del candidato que llegó en segundo lugar.

6 Un total de 18 asambleístas (17,5\% de los 124 que conforman la Asamblea) fueron elegidos por organizaciones conformadas en algunas de las 24 provincias. El sistema electoral ecuatoriano permite presentar candidatos en una sola provincia con el apoyo del 1\% de los inscritos en el padrón electoral de la provincia, lo que abre la puerta para que lleguen a la Asamblea organizaciones minúsculas e incluso prácticamente inexistentes 
Paralelamente, a lo largo del año se produjeron algunas movilizaciones sociales que significaron un quiebre con respecto a los dos primeros años de gestión de Rafael Correa. En efecto, la desmovilización de los grupos sociales que habían sido protagonistas centrales de la política nacional en las dos décadas anteriores fue la característica de esa primera fase. El acceso de fuerzas de izquierda al gobierno -por primera ocasión en el período democrático-, su discurso radical y la calificación del proceso como la revolución ciudadana, fueron los factores determinantes del retroceso de las formas de acción colectiva que caracterizaron a la política ecuatoriana. La apropiación, por parte del gobierno, de las reivindicaciones tradicionalmente impulsadas por estos sectores tuvo mucha importancia en ese sentido. Pero también fue importante el desinterés del gobierno en buscar apoyo en ellos y, en contrapartida, el impulso a una práctica política que colocaba a los movimientos sociales en la disyuntiva de adherirse al gobierno o ser calificados como parte de la oposición o, más aún, de haberse situado en la derecha.

Sin embargo, en diversos momentos del año hubo signos de cambio en este aspecto. De manera especial, la decisión del gobierno en dos áreas de políticas públicas marcó un quiebre que puede considerarse como el inicio de una nueva etapa en la relación con los movimientos sociales. Por un lado, el intento de impulsar una profunda reforma educativa provocó la reacción de la Unión Nacional de Educadores -el sindicato de los maestros de la educación pública-, en tanto que el envío de una ley de recursos hídricos tuvo el mismo efecto sobre las organizaciones indígenas. Los maestros mantuvieron movilizaciones por más de nueve meses, hasta que al finalizar el año obligaron al gobierno a llegar a un acuerdo que les resultaba claramente favorable a ellos. ${ }^{7}$ Por su parte, la CONAIE, que agrupa a las principales organizaciones indígenas del país, convocó a una movilización de alcance nacional y con ello dio muestras de comenzar a salir de la crisis en la que vivió durante los últimos diez años.

Por primera vez en sus casi tres años de gestión, el gobierno debió hacer frente a grupos sociales organizados que impugnaban sus políticas y sobre todo que mostraban capacidad de movilización. La muerte de un indígena amazónico -en un país en donde este tipo de conflictos se ha resuelto tradicionalmente sin violencia- dejó sin sustento a la posición intransigente en que se había colocado el gobierno y lo obligó a abrir el diálogo. Pero, en éste mostró serias limitaciones, expresadas especialmente en la nula apertura para aceptar los planeamientos de las organizaciones sociales y en el subsecuente intento de debilitarlas. ${ }^{8}$

más allá de la contienda electoral. Su condición de representantes de intereses estrictamente acotados y la ausencia de una organización ante la cual ellos deban rendir cuentas les da un amplio margen de acción para negociar puntualmente su apoyo al gobierno y contribuye a la reclusión de la acción política en los niveles subnacionales.

7 La disputa con la UNE significó el alejamiento del Movimiento Popular Democrático, un partido de orientación maoísta, que apoyó al gobierno desde el inicio y que fue un factor determinante en las movilizaciones previas a la convocatoria a la Constituyente y ya en el transcurso de ésta.

$8 \quad$ El gobierno acudió a dos recursos para debilitar a las organizaciones sociales. En primer lugar, desplegó una intensa campaña de medios no sólo para difundir su posición, sino sobre todo para cuestionar la calidad y la integridad de los dirigentes de las organizaciones sociales. Incluso, mientras se desarrollaba la actividad de una comisión conformada para investigar las causas y los responsables de la muerte sucedida en las protestas, emitió piezas comunicacionales en las que sostenía que ésta había sido causada por los propios manifestantes. Por otra parte, buscó fortalecer a organizaciones paralelas, como la Federación Nacional de Organizaciones 
La calificación de estos grupos como conspiradores y contrarrevolucionarios, así como los reiterados intentos de minimizar su peso, su capacidad de movilización e incluso de negar su legitimidad, fueron demostraciones de la ausencia de preparación para una situación en la que las impugnaciones venían desde la izquierda y desde los sectores más pobres. No tenía frente a él a los grupos minúsculos y fragmentados de la oposición parlamentaria, sino a las organizaciones sociales que habían sido protagonistas centrales de la política nacional a lo largo de todo el período democrático. Su convicción de que esos sectores -indígenas, pobres, izquierda- están directamente representados en el gobierno y que por tanto no es posible ni se justifica su protesta, es uno de los elementos que explican la reacción llena de errores, en la que se mezcló indistintamente autoritarismo con paternalismo. ${ }^{9}$

Tres conflictos adicionales afectaron al gobierno. El uno giró en torno al proyecto de ley de comunicación que presentó a la Asamblea, en el que constaban algunos artículos que fueron considerados por varios sectores como atentatorios a la libertad de expresión. De manera especial, se cuestionó el contenido de un artículo que establecía la censura previa y de otro que llevaba a la conformación de un consejo de control de los medios de comunicación en el que tendría mayoría el Ejecutivo. Se abrió así un conflicto que no ha concluido y que abonó al enfrentamiento que el Presidente ha mantenido desde el inicio de su primer mandato con los medios de comunicación. ${ }^{10}$ Paralelamente, el Consejo Nacional de Radio y Televisión abrió tres procesos en contra de un canal de televisión (Teleamazonas), que llevó hasta su clausura temporal, por tres días, en diciembre. ${ }^{11}$

El segundo conflicto surgió cuando el Presidente cuestionó al equipo gubernamental, encabezado por su canciller, que negociaba con diversos países la entrega de una compensación económica a cambio de mantener sin explotar un campo petrolero situado dentro de un área de gran biodiversidad. ${ }^{12}$ El cuestionamiento -realizado en su programa semanal de radio y televisión sin tratamiento previo en el gabinete- provocó la salida del canciller, el desmantelamiento del equipo técnico-político y el estancamiento de las negociaciones.

Campesinas Indígenas y Negras (FENOCIN), de filiación evangélica, con la que mantuvo una alianza hasta mayo de 2010 (después del período analizado en este texto). Al ser una organización rival de la CONAIE, ésta se constituyó en factor ideal para neutralizar al movimiento indígena.

9 El conflicto con los maestros terminó con un acuerdo que significó la aceptación de prácticamente todos los puntos planteados por ellos, lo que traería consecuencias negativas para el proceso de reforma educativa impulsado por el gobierno. Por otra parte, para solucionar el conflicto con los indígenas se acudió a la firma de un acuerdo que, al estar planteado en términos extremadamente generales, no tuvo expresión práctica (lo que explica en gran medida la reaparición, y con mayor fuerza, de las movilizaciones en los primeros meses del año 2010).

10 El presidente Correa ha calificado reiteradamente a los medios como "prensa corrupta" y los ha acusado de actividades conspirativas y de defender intereses de grandes grupos económicos. En la posesión para su segundo mandato, en agosto de 2009, calificó a los medios de comunicación como el principal enemigo de su gobierno y de la revolución ciudadana. En su programa de los sábados mantiene un segmento denominado "la libertad de expresión ya es de todos", en el que pasa revista a la manera en que los medios han tratado, desde la información o desde la opinión, los hechos relacionados con el gobierno.

11 Como hechos curiosos y anecdóticos, cabe señalar que una de las acciones emprendidas en contra de ese canal se debió al aparecimiento de una corrida de toros, por menos de diez segundos, dentro de la cobertura de las festividades de una ciudad y, paralelamente, se pretendía censurar la serie norteamericana Los Simpson.

12 La renuncia del canciller se produjo el 12 de enero de 2010, pero, el conflicto se inició en diciembre de 2009, cuando el Presidente impidió que la delegación ecuatoriana a la reunión de Copenhague sobre cambio climático presentara la propuesta de no explotación del petróleo, denominada ITT-Yasuní. 
Además, marcó la profundización de las diferencias que venían arrastrándose desde la Asamblea Constituyente, con los sectores de izquierda dentro de Alianza País. ${ }^{13}$ Con ello, ganaron espacio los otros grupos que integran el gobierno, especialmente quienes han llegado a sus cargos por la amistad con el Presidente y los que provienen de una trayectoria más técnica que política o ideológica.

El tercer problema se presentó en el mes de junio, cuando un medio de comunicación dio a conocer que el hermano del Presidente mantenía contratos con el Estado por un monto cercano a los cien millones de dólares. ${ }^{14}$ Este episodio afectó al gobierno en uno de los aspectos más sensibles, ya que ponía en duda su proclama de luchar contra la corrupción, más aún cuando ocurría en el entorno familiar del Presidente. Pero, a pesar de que la opinión pública -expresada en los medios de comunicación- fue abiertamente crítica con la reacción presidencial, no ocurrió lo mismo con el apoyo -expresado en encuestas- que se mantuvo prácticamente inalterado (Serviquanti-Habitus, 2009).

\section{Coyuntura económica}

En el campo económico, el año 2009 continuó la tendencia establecida en el año anterior, con bajos índices de crecimiento e inversión. El crecimiento de la economía fue negativo, de $-0,6 \%$, constituyéndose en el tercer año consecutivo de decrecimiento (BCE, 2010). ${ }^{15}$ Por consiguiente, ese deficiente desempeño no puede ser atribuido en su totalidad o exclusivamente a la crisis económica mundial. Ésta influyó, sin duda, pero no puede ser considerada como la causa del bajo crecimiento que, en términos de producto por habitante (PIB per cápita), significó un descenso desde US\$3.961 en el año 2008 a US\$3.669 en el año 2009, equivalente a $-7,4 \%$ (BCE, 2010). Es probable que las causas últimas se encuentren en las medidas tomadas para impulsar el cambio del modelo económico, que ha sido la propuesta del gobierno desde la campaña electoral y que se ha traducido en una mayor y decisiva presencia estatal en la economía. El incremento del gasto público, especialmente en áreas como infraestructura vial, educación y salud, ha sido visto por

13 El presidente Correa cuestionó con dureza la constitución de un fideicomiso para la administración de los recursos que se obtendrían a cambio de no explotar el petróleo, por considerar que era un atentado a la soberanía nacional. Además, calificó como ecologistas infantiles a quienes identificó como los autores de la propuesta, incluido su propio canciller (Diario El Universo, 15 de enero de 2010). El enfrentamiento con ese sector permitió ver que el problema de fondo no se encontraba en la modalidad del fideicomiso, sino que era la disputa entre un modelo ecologista o ambientalista, defendido por los ecologistas infantiles, y uno de carácter extractivo-primario, que impulsa el Presidente (Diario El Universo, 25 de enero de 2010).

14 La investigación periodística fue realizada por el diario Expreso y difundida en varias entregas a partir del 7 de junio. Las reacciones del Presidente fueron contradictorias y cambiantes, ya que inicialmente sostuvo que no existía ilegalidad, que tampoco se habían causado perjuicios al Estado y que se trataba de una persecución a su familia con el propósito de afectarle a él (Diario Expreso, 15 de junio de 2009). Más adelante, cuando esa posición se hizo insostenible a causa de la contundencia de la información y cuando se generalizaba una opinión que calificaba a éste como un caso de tráfico de influencias, Rafael Correa aludió al aspecto ético y condenó la actitud de su hermano, pero sostuvo que desconocía la existencia de los contratos (El Universo, 21 de junio de 2009).

15 Las cifras del Banco Central, utilizadas en este trabajo, no coinciden con las de otras fuentes nacionales ni con las de los organismos internacionales. Así, el Ministerio de Finanzas establece un crecimiento de 0,41\%, la CEPAL de -0,4\%, el Fondo Monetario Internacional de -1,0\% y el Banco Mundial de -2,3 (Acosta et al., 2010: 46) De cualquier manera, solamente el Ministerio de Finanzas adjudica un crecimiento positivo. 
algunos economistas como una de las explicaciones de ese comportamiento de las variables económicas (Oleas, 2009: 4).

Por otra parte, el gobierno logró una reducción significativa de la deuda externa, aunque el procedimiento utilizado planteó fuertes riesgos que aún no han sido despejados en su totalidad. En lugar de una negociación con los portadores de los bonos, declaró unilateralmente el desconocimiento de un tramo de la deuda, que sumaba aproximadamente US\$ 3.000 millones. ${ }^{16}$ De esta manera, la deuda pública externa bajó a US\$ 7.364 en el año 2009, después de que había sido de US\$ 10.605 en el año 2007 y de US\$ 10.028 en el año 2008. Esto colocó a la deuda pública externa en el 14,3\% del PIB, después de haber representado el 23,2\% y el 18,3\%, en 2007 y 2008, respectivamente. Por tanto, la negociación del gobierno acentuó la tendencia al descenso del peso de la deuda externa y, aunque la interna se mantuvo en niveles que bordean los tres mil millones de dólares, alivió sustancialmente la presión sobre el presupuesto del Estado y en general sobre la economía. Pero, hacia los últimos meses del año se hizo evidente que esas decisiones tuvieron efectos negativos, especialmente por el cierre de las fuentes de financiamiento externo, por lo que el gobierno debió acudir a un préstamo muy oneroso por parte de China. ${ }^{17}$

Un factor que contribuyó a evitar un mayor decrecimiento de la economía fue el alto precio del petróleo. La media del año fue de 60,1 dólares por barril, mientras en diciembre de 2008 se había situado en 26 dólares. Sin embargo, el incremento de precios en el mercado internacional no tuvo una respuesta similar en la explotación y en la exportación, lo que se manifestó en el decrecimiento de - $0,6 \%$ del PIB petrolero en el año 2009 y en la caída de las exportaciones desde US\$ 11.673 millones, en 2008, hasta US\$ 6.965 millones, en 2009 (Carta Económica, 2010-4: 8). Adicionalmente, los altos precios del petróleo facilitaron el retorno a las épocas en que la economía dependía mayoritariamente de este producto, lo que a su vez -por el impulso a las prácticas rentistas- se transformó en un aliciente para la permanencia de una economía basada en actividades primarias y extractivas. Las expectativas de impulsar una reconversión económica, que había albergado el gobierno en su plan de desarrollo, se diluyeron en la bonanza petrolera.

Finalmente, el bajo desempeño de la economía se reflejó en el incremento del desempleo y del subempleo, así como en la ruptura de la tendencia a la baja de la incidencia de la pobreza. En efecto, el desempleo subió de 7,3\%, en diciembre de 2008, a 9,1\% en septiembre

16 La compra de deuda en mercados secundarios y por medio de terceras personas -al margen de las normas establecidas para este tipo de transacciones- dificulta el establecimiento del monto final de la deuda no reconocida al ser calificada como ilegítima por el gobierno ecuatoriano. La ministra de Finanzas aludió a un monto de 7.505 millones de dólares (Diario Hoy, 3 de enero de 2010), pero las cifras del Banco Central reflejan 3.486 millones (BC, 2010). El anuncio de la decisión gubernamental fue hecho por el presidente Correa el 11 de junio de 2009 (Diario Expreso, 12 de junio de 2009).

17 El gobierno ecuatoriano obtuvo un préstamo de mil millones de dólares bajo la forma de venta anticipada de petróleo a China. Ecuador se comprometió a entregar 96 mil barriles diarios durante dos años, a US\$ 56 por barril (por debajo del precio medio del crudo ecuatoriano), con un interés adicional de 7,25\%, lo que le convirtió en la deuda más cara en la historia del país. El monto final a pagar será de US\$ 3.924 millones (Diario Hoy, 28 de julio de 2009). 
de 2009 y a 7,9\% en diciembre de 2009 (INEC, 2009: diciembre). ${ }^{18}$ Por su parte, el subempleo se incrementó desde el 48,8\% de la población económicamente activa, en diciembre de 2008, hasta el 50,5\%, en diciembre de 2009. Previamente había llegado al 51,9\% en enero, el 51,6\% en junio, y el 51,7\% en septiembre. Por consiguiente, entre los desempleados y los subempleados alcanzaron el 58,4\% de la PEA en diciembre de 2009 .

La incidencia de la pobreza mantuvo una tendencia decreciente desde el año 2003, cuando se redujo de $49,1 \%$ de la población, al $43,8 \%$ en 2004 , al $41,9 \%$ en 2005 , al 37,4\% en 2006, al 36,6\% en 2007 y al 35,1\% en 2008 (INEC, 2009: diciembre). Por tanto, en cinco años bajó 14 puntos porcentuales, equivalentes casi a un tercio del nivel inicial. Sin embargo, en el año 2009 tuvo un pequeño incremento al situarse en 35,8\%, lo que puede ser un indicador del fin de aquella tendencia o por lo menos de su moderación. De cualquier manera, resulta sorprendente que este sea el resultado de la gestión de un gobierno que ha emprendido un conjunto de programas y proyectos dirigidos a atender las necesidades de los sectores más pobres y que ha definido a la redistribución del ingreso como una de sus metas. ${ }^{19}$

\section{Coyuntura internacional}

En el plano internacional, el gobierno de Rafael Correa decidió la integración de Ecuador como socio pleno de la Alianza Bolivariana para los Pueblos de Nuestra América, ALBA. Hasta junio del año 2009 solamente había participado como observador, manteniendo una relativa distancia con la iniciativa del presidente venezolano Hugo Chávez. Eso le permitió administrar sus relaciones internacionales desde una posición equidistante con los diversos bloques ideológicos que se han conformado, formal o informalmente, entre los países latinoamericanos. El ingreso a esta asociación, por el contrario, tiende a alinear al país en uno de los grupos, lo que sin duda traerá diversas consecuencias en el plano de su inserción internacional. Adicionalmente, la firma de un convenio entre el Banco Central del Ecuador con un banco estatal de Irán, sobre el que pesaban sanciones de Naciones Unidas y de varios países por la acusación de financiar actividades terroristas, produjo -a inicios del año 2010- la sanción a Ecuador por parte del Grupo de Acción Financiera Internacional, GAFI (Financial Action Task Force), un organismo de control del financiamiento del terrorismo, creado por el G7 en 1989 (Diario Expreso, 19 de febrero de 2010).

A lo largo del año se mantuvo la tensión con el gobierno colombiano, sin que se llegaran a restablecer las relaciones que habían sido rotas en marzo de 2008. La incursión de las tropas colombianas en territorio ecuatoriano donde se encontraba el campamento del segundo hombre de las FARC, Raúl Reyes, produjo aquella ruptura y derivó en un clima de tensión que se escenificó en varios foros internacionales. Desde la presidencia

18 El Instituto Ecuatoriano de Estadísticas y Censos, INEC, encargado de la medición del empleo, entrega trimestralmente las cifras, de manera que no es posible conocer la evolución mensual. Asimismo, no es posible comparar con los años anteriores a 2007, porque en ese año se cambió la metodología de medición.

19 La distribución del ingreso muestra un leve avance, ya que al medírsela por el coeficiente de Gini se redujo de 0,580 en el año 2000 a 0,499 en el año 2009, habiendo tenido un incremento hasta 0,600 en el año 2001. 
pro témpore de UNASUR, que asumió en el mes de agosto, Rafael Correa calificó a la instalación de bases norteamericanas en territorio colombiano como un atentado a la seguridad regional e impulsó medidas de control que finalmente no fueron aprobadas en la reunión desarrollada en Bariloche, Argentina (Diario El Universo, 30 de agosto de 2009).

\section{ELECCIONES Y REACOMODO DE FUERZAS POLÍTICAS}

En abril del año 2009 se realizaron las elecciones generales, las primeras dentro de la Constitución aprobada en noviembre de 2008. Estas se produjeron en un contexto caracterizado por la crisis de los partidos que habían predominado a lo largo de los treinta años del período democrático, el cambio institucional derivado del nuevo ordenamiento constitucional y el predominio del fuerte liderazgo de Rafael Correa.

En la elección presidencial se destacó, como hecho extraordinario, el triunfo de un candidato en primera vuelta con la mayoría absoluta de los votos. Desde la instauración del balotaje, en 1978, siempre había sido necesaria la realización de la segunda vuelta e incluso en tres ocasiones se produjo la reversión del resultado de la primera vuelta (la última de ellas precisamente en la primera elección de Rafael Correa, en el 2006). La debilidad de las candidaturas presidenciales fue la tónica a lo largo de todo ese período, hasta el punto que la media de quienes ocuparon el primer puesto en la primera vuelta, entre 1978 y 2006, fue de $27,8 \%$, con el nivel más alto de $34,9 \%$ en $1988 .{ }^{20}$ Incluso en el año 2006, en la primera elección en que participó Rafael Correa (cuando ocupó el segundo lugar en la primera vuelta), el triunfador obtuvo solamente el $26,8 \%$ de los votos. La fragmentación del sistema de partidos y la volatilidad electoral fueron factores determinantes para llegar a esos resultados. En esta ocasión, por el contrario, la votación se concentró en la candidatura del presidente que buscaba la reelección después de veintisiete meses de gestión. Así, Rafael Correa obtuvo el 52,0\% de los votos, a una distancia de 25,2 puntos porcentuales de su competidor más cercano, como se puede ver en el Cuadro 1.

Sin embargo, esa votación no alcanzó los niveles logrados en las dos consultas populares convocadas para definir la instalación de la Asamblea Constituyente (de septiembre de 2007, en que su propuesta obtuvo el $82 \%$ ) y para aprobar la Constitución (en septiembre de 2008, en que alcanzó el 64,4\%). ${ }^{21}$ Asimismo, en la elección de asambleístas (para el órgano legislativo denominado Asamblea Nacional en la nueva Constitución) Alianza País obtuvo el 43,5\% de los escaños, lo que significó un descenso de su votación con respecto a la elección de asambleístas constituyentes de septiembre de 2007, que fue de 56,2\% de los escaños. Por consiguiente, a pesar de la distancia con los demás partidos o agrupaciones políticas (que se puede apreciar en el Cuadro 2), la elección legislativa significó hasta

20 Todas las cifras electorales están tomadas de la página web del Consejo Nacional Electoral (anteriormente Tribunal Supremo Electoral).

21 Ciertamente, no son comparables los procesos de consulta o referéndum con las elecciones presidenciales, pero sí se puede tomar a las primeras como una referencia general del respaldo obtenido por Rafael Correa a lo largo de su gestión. 
Cuadro 1: Elección presidencial. 2009

\begin{tabular}{lrc}
\hline \multicolumn{1}{c}{ Candidato } & \multicolumn{1}{c}{ Votos } & Porcentajes \\
\hline Rafael Correa & 3.586 .439 & 52,0 \\
Lucio Gutiérrez & 1.947 .830 & 28,2 \\
Álvaro Noboa & 786.718 & 11,4 \\
Martha Roldós & 298.765 & 4,3 \\
Carlos Sagnay & 108.079 & 1,6 \\
Melba Jácome & 93.146 & 1,4 \\
Diego Delgado & 43.221 & 0,6 \\
Carlos González & 33.714 & 0,5 \\
Total & 6.897 .912 & 100,0 \\
\hline
\end{tabular}

Fuente: Consejo Nacional Electoral. Elaboración: propia.

Cuadro 2: Asambleístas según partido político. 2009

\begin{tabular}{lcc}
\hline \multicolumn{1}{c}{ Partido } & Escaños & Porcentajes \\
\hline AP & 54 & 43,5 \\
PSP & 18 & 14,5 \\
MOVS. PROVINC. & 18 & 14,5 \\
MADERA & 7 & 5,6 \\
PRIAN & 6 & 4,8 \\
PSC & 4 & 3,2 \\
MPD & 4 & 3,2 \\
PRE & 3 & 2,4 \\
PAIS-MED & 3 & 2,4 \\
MUPP & 3 & 2,4 \\
ID & 2 & 1,6 \\
PAIS-PS-FA & 2 & 1,6 \\
Total & 124 & 100,0 \\
\hline
\end{tabular}

Fuente: Consejo Nacional Electoral. Elaboración: propia.

cierto punto un revés para AP y en general para el proyecto de la revolución ciudadana, ya que perdió el enorme margen de acción que tuvo en la constituyente y, en consecuencia, se vio en la necesidad de establecer acuerdos con otras fuerzas políticas. ${ }^{22}$

22 La diferencia entre las dos situaciones se hace evidente en el número efectivo de partidos (medido de acuerdo al índice de Laakso y Taagepera) que subió de 2,9 en la Asamblea Constituyente a 4,1 en la Asamblea Nacional. Aunque el índice actual resulta relativamente bajo en relación a los niveles alcanzados en el Congreso Nacional a lo largo de los treinta años anteriores (en que la media del número efectivo fue de 7,7), podría interpretarse como el inicio del retorno hacia las condiciones anteriores (cabe destacar que se sitúa prácticamente en el mismo nivel que tuvo en el último Congreso, en el año 2006, que fue de 4,2). 
Gráfico 1: Asamblea Nacional: escaños por partido. 2009

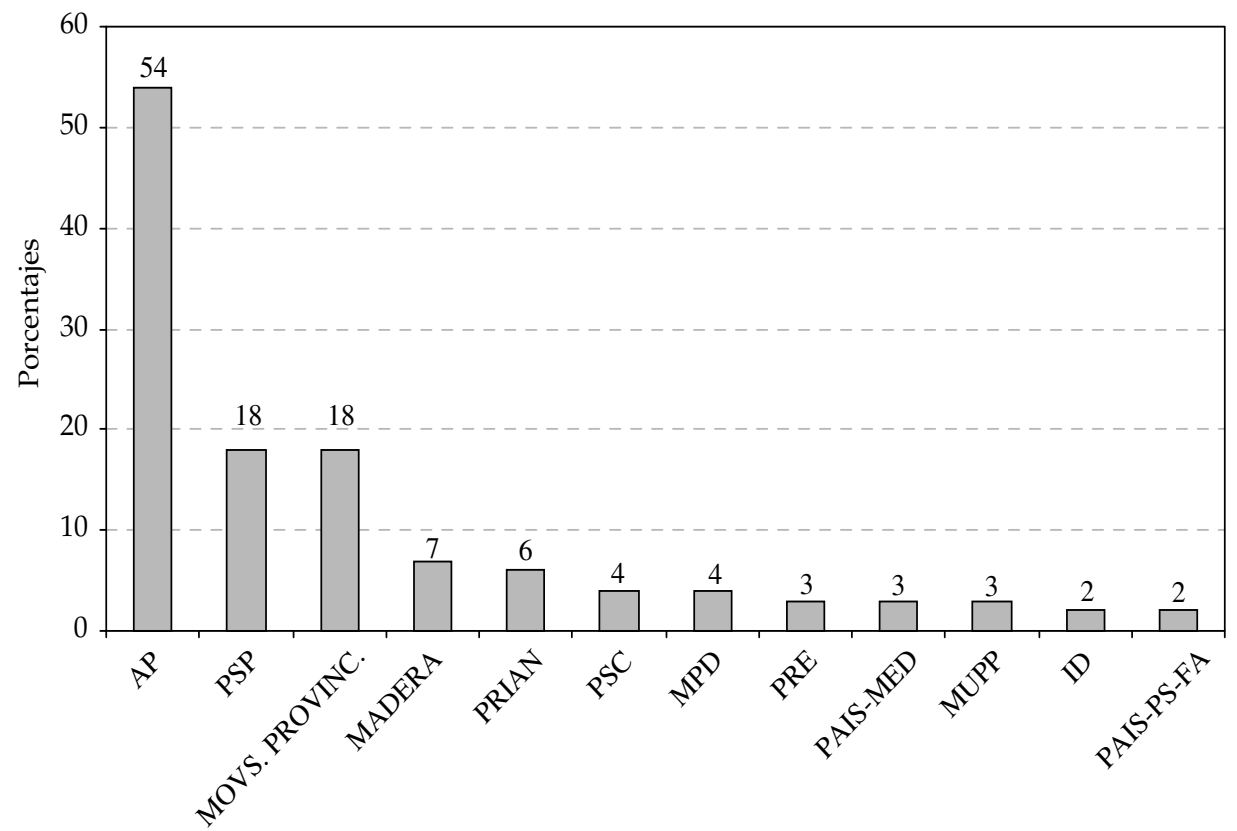

Como ha ocurrido tradicionalmente en la política ecuatoriana, el partido gubernamental encontró el apoyo necesario en los movimientos de origen provincial, que requieren del gobierno para llevar adelante sus propuestas de carácter fundamentalmente local (Pachano, 2008; Freidenberg, 2010). De esta manera, los grupos con menor definición ideológica y organizados casi exclusivamente con fines electorales pasaron a convertirse en elementos fundamentales para el desarrollo del proyecto de la revolución ciudadana. El fuerte liderazgo del presidente Correa pasó a convertirse en el único factor que podía garantizar la continuidad del proyecto, con todos los riesgos que conlleva hipotecarlo a una sola persona.

En las elecciones de organismos provinciales, AP tuvo un desempeño bastante favorable al obtener 9 de las 23 prefecturas provinciales (39,1\%). También obtuvo un buen resultado en la elección de alcaldes municipales, cuando logró el control de 71 municipios de los 221 existentes en el país $(32,1 \%) .{ }^{23}$ Sin embargo, son cifras que guardan mucha distancia con el apoyo obtenido por el presidente de la República e incluso con la proporción de escaños obtenida en la Asamblea Nacional. Paralelamente, es importante destacar la votación obtenida por los movimientos de origen provincial y/o local, que se situaron en el segundo lugar en las municipales y en cuarto lugar en las de prefectos provinciales (Cuadros 3 y 4 ). 
Cuadro 3: Prefectos provinciales según partido político. 2009

\begin{tabular}{lcc}
\hline \multicolumn{1}{c}{ Partido } & Prefectos & Porcentajes \\
\hline Alianza País & 9 & 39,1 \\
Partido Sociedad Patriótica & 4 & 17,4 \\
Pachakutik & 4 & 17,4 \\
Movimientos provinciales & 3 & 13,0 \\
Mov. de Integración Nacional & 2 & 8,7 \\
Movimiento Popular Democrático & 1 & 4,3 \\
Total & 23 & 100,0 \\
\hline
\end{tabular}

Fuente: Consejo Nacional Electoral. Elaboración: propia.

Cuadro 4: Alcaldes municipales según partido político. 2009

\begin{tabular}{lcc}
\hline \multicolumn{1}{c}{ Partido } & Alcaldes & Porcentajes \\
\hline Alianza País & 71 & 32,1 \\
Movimientos locales & 38 & 17,2 \\
Pachakutik & 35 & 15,8 \\
Partido Sociedad Patriótica & 30 & 13,6 \\
Alianzas locales & 27 & 12,2 \\
Mov. de Integración Nacional & 20 & 9,0 \\
Total & 221 & 100,0 \\
\hline
\end{tabular}

Fuente: Consejo Nacional Electoral. Elaboración: propia.

Asimismo es significativa la posición ocupada por Pachakutik, el partido originado en el movimiento indígena, con cuatro prefecturas y 35 alcaldías municipales.

Cuatro elementos centrales se desprenden de los resultados de este conjunto de elecciones. En primer lugar, la crisis de los partidos tradicionales, puesta en evidencia desde las elecciones del año 2002, parece haber alcanzado su expresión final. Los partidos que predominaron a lo largo de las tres décadas previas (Social Cristiano, Izquierda Democrática, Roldosista Ecuatoriano y Democracia Popular-Unión Demócrata Cristiana) prácticamente han desaparecido del escenario político nacional. Su presencia en la Asamblea es exigua y se reduce a 9 escaños (que llegan a 16 si se añaden los 7 del movimiento Madera de Guerrero, que se constituyó a partir del PSC). Aunque es un mejor resultado que el que obtuvieron para la Constituyente, en que sumaron siete escaños, es innegable -y seguramente irreversiblela pérdida de su condición de actores centrales del sistema de partidos.

En segundo lugar, los dos partidos que aparecían en la elección de 2006 como los posibles reemplazos de los tradicionales, obtuvieron resultados muy diferentes. Sociedad Patriótica, PSP, se situó en el segundo lugar, con 18 escaños, en tanto que el Partido Renovador Independiente de Acción Nacional, PRIAN (que disputó la segunda vuelta del año 2006 con su candidato Álvaro Noboa), ocupó el quinto lugar con seis escaños. Ambos se situaron en 
niveles muy cercanos a los que tuvieron en la Asamblea Constituyente, que fueron de 18 y 8 , respectivamente (aunque algo más bajos en términos proporcionales por el diferente número de integrantes de cada organismo). Con ello, se ve muy lejana la posibilidad de transformarse en los ejes de un nuevo sistema de partidos, sobre todo en el caso del PRIAN que además mostró una fuerte concentración territorial de su votación.

En tercer lugar, a pesar del descenso en la votación y en el número de escaños, AP se mantuvo como el partido hegemónico en estas elecciones. Como lo han destacado varios autores (Freidenberg, 2010; López, 2009), esta es una situación desconocida en un país que se ha caracterizado por la fragmentación de la representación política y por la debilidad de los partidos. Del mismo modo, el número de escaños obtenidos por AP marca un quiebre con la tradicional presencia de gobiernos de minoría. Aunque no alcanzó la mayoría absoluta, no resulta despreciable la proporción alcanzada, que lo coloca en una situación muy ventajosa para establecer alianzas. Sin embargo, como se señaló antes, por un conjunto de factores éstas han debido hacerse con los movimientos provinciales o con partidos de tendencias ideológicas muy diferentes a las suyas y en general distantes de la propuesta de la revolución ciudadana. Los problemas derivados de esto se han manifestado en las dificultades para el procesamiento de las leyes establecidas como prioritarias y de tratamiento obligatorio por la Constitución y que son vitales para el proyecto político de AP.

Por último, en estas elecciones se han expresado al mismo tiempo dos tendencias de signo contrario en términos de la distribución territorial de la votación. La primera, tendiente a la distribución armónica en el territorio nacional, se expresa en la votación de AP y PSP. La segunda es la de los partidos y organizaciones que tienden a concentrar su votación en una sola provincia o, en el mejor de los casos, en una sola región del país, y se expresa tanto en partidos que cuentan con varios años de existencia (como el PRIAN y el PRE), como en las organizaciones de alcance provincial que generalmente se estructuran en exclusivo para una elección. Esta últimas se benefician de las disposiciones legales que facultan a las organizaciones políticas a presentar candidatos en una sola provincia, sin necesidad de contar con una organización de alcance nacional.

\section{PODERES DEL ESTADO: DESEQUILIBRIO Y EXPERIMENTOS}

\section{Desequilibrio y debilidad de controles}

Diversas reformas constitucionales, realizadas a lo largo de los últimos treinta años, profundizaron el carácter presidencial del régimen político ecuatoriano (Pachano, 2007). En el diseño de la Constitución de 1998 se buscó incrementar los umbrales de gobernabilidad por medio de la limitación de funciones del Congreso Nacional y el fortalecimiento del Ejecutivo. La Constitución del año 2008 profundizó esa tendencia al despojar de la mayor parte de funciones al órgano legislativo. Su capacidad de control a los otros poderes del Estado y la participación en el nombramiento de autoridades de otras funciones fueron reducidas al mínimo. Incluso su participación en la aprobación del presupuesto del Estado se redujo de manera significativa (Constitución Política, artículo 120-12). La mayor parte de esas funciones fueron trasladadas a un órgano denominado 
Consejo de Participación Ciudadana y Control Social, CPCCS que, conjuntamente con la Defensoría del Pueblo, la Contraloría y las superintendencias conforman el denominado quinto poder del Estado. ${ }^{24}$

Tanto con las últimas reformas como con las anteriores se buscó incrementar los poderes constitucionales del Ejecutivo, pero no se dieron pasos para que se incrementaran también sus poderes políticos. Particularmente, se dejó intocado el sistema electoral (basado en la votación por personas en listas abiertas), que contiene enormes incentivos para la fragmentación y para la volatilidad. Los resultados de esta omisión fueron evidentes en los resultados electorales reseñados en la sección anterior, especialmente en el alto número de listas que obtuvieron representación legislativa (un total de 19). Por consiguiente, se ha mantenido y se ha ahondado la contradicción, señalada por Shugart y Carey (1992), entre un Ejecutivo que dispone de fuertes poderes constitucionales pero que a la vez es extremadamente débil en términos políticos.

Esa ha sido una de las características centrales del sistema político ecuatoriano, que en esta ocasión no se ha manifestado en toda su magnitud porque el fuerte liderazgo del presidente Correa actúa como un elemento de compensación de la debilidad política. Por tanto, la fortaleza política del Ejecutivo no descansa en factores institucionales sino en las condiciones pasajeras del liderazgo presidencial, lo que genera un tipo de incertidumbre diferente a la que predominó a lo largo de los últimos quince años, pero tan nociva para la gobernabilidad y la estabilidad como ella. Los ya mencionados problemas en el procesamiento de las leyes establecidas constitucionalmente son sus expresiones más visibles, aunque no las únicas.

Adicionalmente a esas características institucionales, desde el inicio de sus actividades en agosto de 2009 la Asamblea Nacional ha funcionado con escasa autonomía, cediendo la iniciativa de la legislación al Ejecutivo. Como se puede ver en el Cuadro 5, de los 148 proyectos de ley presentados en la Asamblea, $23(15,5 \%)$ fueron de iniciativa del presidente de la República y 119 (80,4\%) de diversos asambleístas, pero solamente 14 de estos últimos tomaron la forma definitiva de ley, mientras que 12 de los que se originaron en la Presidencia cumplieron ese ciclo. En términos de eficacia (medida por la proporción de proyectos aprobados sobre el total de presentados) esto significa que la Asamblea tuvo un $11,8 \%$, frente a un $52,2 \%$ del Ejecutivo. Es evidente la preferencia asignada a las iniciativas gubernamentales, que se explica en gran medida porque desde allí surgieron las leyes establecidas como prioritarias en la Constitución. La Asamblea no asumió esa responsabilidad, como pudo hacerlo ya que no existe una disposición constitucional o legal que atribuya esa iniciativa al Ejecutivo. por medio de un concurso de méritos, tiene entre sus atribuciones el nombramiento de las autoridades de organismos como la Contraloría, las superintendencias, la Defensoría del Pueblo, la Fiscalía, la Corte de Justicia, la Defensoría Pública, la Procuraduría, el Consejo Nacional Electoral, el Tribunal Contencioso Electoral y el Consejo de la Judicatura. 
Cuadro 5: Proyectos de ley según estado del trámite en la Asamblea Nacional

\begin{tabular}{lrrrrr}
\hline \multirow{2}{*}{ Iniciativa } & \multicolumn{5}{c}{ Estado del trámite } \\
\cline { 2 - 6 } & Presentadas & $\begin{array}{c}\text { Primer } \\
\text { debate }\end{array}$ & $\begin{array}{c}\text { Segundo } \\
\text { debate }\end{array}$ & Aprobadas & $\begin{array}{c}\text { Sin } \\
\text { tramitar }\end{array}$ \\
\hline Presidente de la República & 23 & 4 & 3 & 12 & 4 \\
Asambleístas & 119 & 11 & 4 & 14 & 90 \\
Otros & 6 & 1 & & 2 & 3 \\
Total & 148 & 16 & 7 & 28 & 97 \\
\hline
\end{tabular}

Fuente: Asamblea Nacional: www.asambleanacional.gov.ec/tramite-de-las-leyes.html

\section{De las cuotas al gabinete monocolor}

La práctica usual para enfrentar la ausencia de mayoría en el Legislativo fue la conformación de alianzas, ya sean abiertas o fantasmas, así como la integración en el gabinete de los partidos que participaban en ellas (Mejía, 2009: 42-61). Así se podía superar en alguna medida los problemas de debilidad política del Ejecutivo, especialmente en su relación con el Legislativo. Sin embargo, el gobierno de Rafael Correa no tomó ese camino y optó más bien por conformar gabinetes seleccionados de acuerdo a criterios ideológicos, técnicos y de fidelidad a su proyecto político. Por ello, desde el inicio del gobierno el gabinete ha expresado una combinación de militantes de su movimiento político, tecnócratas y personas cercanas al Presidente (Basabe, 2009: 13). En ningún momento ha incluido a personas provenientes de otros partidos políticos ni de organizaciones sociales, lo que puede interpretarse como una forma de perseverar en la modalidad utilizada a lo largo de su primer mandato, cuando contaba con la mayoría absoluta en la Asamblea Constituyente. ${ }^{25}$ En aquel momento no era imperativo compartir la gestión gubernamental con otros sectores, pero se podía suponer que al perder la mayoría en el Legislativo el gobierno revisaría su posición de indiferencia hacia otras fuerzas políticas y sociales. La continuación del gobierno en solitario puede ser una señal de la enorme confianza depositada en el liderazgo

25 Sorprendentemente -ya que va en sentido contrario al enfrentamiento con lo que él denomina partidocraciadentro de esa conformación monocolor del gabinete, el presidente Correa ha incluido a varias personas que colaboraron con anteriores gobiernos o que incluso hicieron trayectoria política dentro de otros partidos políticos. Ellas han sido parte de la cuota tecnocrática, a pesar de que ocupan cargos de responsabilidad política. Así, Carlos Vallejo, ex militante demócrata cristiano, ministro en varios gobiernos y diputado por el PRIAN, ha ocupado en el presente gobierno el ministerio de Agricultura y la presidencia del Banco Central; Diego Borja, actual presidente del Banco Central, y hasta abril de 2010 ministro de Coordinación Económica, fue ministro de Economía en el gobierno de Alfredo Palacio y asambleísta constituyente por la ID; Nathalie Cely fue ministra de Economía con Jamil Mahuad y actualmente ocupa el mismo cargo; Raúl Vallejo, ministro de Educación durante toda la gestión de Rafael Correa (hasta abril de 2010), ocupó el mismo cargo en los gobiernos de Rodrigo Borja y de Alfredo Palacio; Ramiro González, Director del Instituto Ecuatoriano de Seguridad Social, fue candidato por Izquierda Democrática a la vicepresidencia de la República en el año 2006 (compitiendo contra la fórmula de Correa) y ocupó por dos períodos la prefectura de la provincia de Pichincha en representación de su partido; Doris Soliz, ministra de Coordinación de Patrimonio Nacional y Cultural (y de Coordinación Política desde abril de 2010), fue ministra de Turismo en el gobierno de Lucio Gutiérrez. 
presidencial, pero también de una incorrecta evaluación de la situación inaugurada con las elecciones de abril de 2009.

Como ha ocurrido en gobiernos anteriores, el actual ha buscado mantener el equilibrio regional en el gabinete. El origen provincial y regional de sus integrantes es uno de los criterios que se toman en cuenta en el momento de su conformación, lo que se explica por el fuerte peso del clivaje regional en la política ecuatoriana (Pachano, 1985; 2008, Freidenberg y Alcántara, 2001). Por otra parte, a lo largo del año 2009 se mantuvo la distribución de género que se mantenía desde el inicio, con aproximadamente $60 \%$ de hombres y $40 \%$ de mujeres. ${ }^{26}$

Un hecho que sobresalió dentro de la relación entre los poderes del Estado fue el inicio del proceso para seleccionar a los miembros del Consejo de Participación Ciudadana y Control Social. ${ }^{27}$ Como se señaló antes, los integrantes de este órgano se escogen por medio de un concurso de merecimientos y oposición, de manera que ellos no expresan la voluntad de la ciudadanía. Adicionalmente, el reglamento con el que fue convocado el concurso (derivado de la Ley Orgánica de Participación) incluyó la participación política previa y el desempeño de cargos de elección (en los dos años anteriores) como prohibición para presentarse a éste. Por tanto, se castigó a quienes contaban con alguna experiencia política y en general a las personas que habían triunfado en elecciones, lo que en la práctica significa sancionar la experiencia y poner obstáculos para la carrera política. El objetivo de estas medidas era una supuesta despolitización del Consejo, lo que resulta poco menos que incomprensible cuando se trata de una de las funciones del Estado.

El proceso de selección fue cuestionado por parte de diversos sectores que destacaron irregularidades y falta de transparencia en el concurso (Diario Hoy, 25 de enero de 2010; Diario Expreso, 16 de marzo de 2010).$^{28} \mathrm{El}$ Consejo Nacional Electoral, encargado de este proceso, no pudo desvirtuar la mayor parte de esas acusaciones, lo que cubrió de dudas a los resultados finales. Estas se acrecentaron cuando se conocieron los resultados, ya que se demostró que cinco de los siete seleccionados tenían nexos políticos, tres de ellos con el partido de gobierno, uno con un partido aliado y uno con un partido de oposición (Diario El Universo, 7 de febrero de 2010).

26 En el cambio de gabinete realizado en abril de 2010, el Presidente sostuvo que hasta ese momento "ha habido 60 por ciento hombres y 40 por ciento mujeres, probablemente en el nuevo gabinete haya 50-50, o tal vez más mujeres" (Diario Hoy, 22 de abril de 2010).

27 El 15 de marzo de 2010 concluyó el proceso de conformación del CPCCS, pero la convocatoria para el concurso se realizó en julio de 2009, de manera que la mayor parte del proceso ocurrió en ese año.

28 Los postulantes debían realizar un examen en el que algunas preguntas estaban planteadas en términos de respuestas únicas pero en realidad podían ser múltiples, como cuando se consultaba si en el Ecuador existe a) monarquía constitucional, b) parlamentarismo, c) presidencialismo; d) federalismo. En otros casos las preguntas tenían alta dosis de imprecisión, como cuando se consultaba cuál de las siguientes organizaciones es un movimiento social: a) Cámara de Industrias de Pichincha, b) Consejo Nacional de Mujeres; c) Confederación de Jubilados y Pensionistas; d) Consejo Nacional de Discapacidades (Diario Hoy, 9 de enero de 2009). 


\section{La dimensión subnacional}

Como se ha mencionado antes, el clivaje regional no puede ser soslayado en un análisis de la política ecuatoriana. La existencia de espacios en los que se conforman sociedades regionales con fuertes identidades territoriales (Pachano, 2006) constituye la base sobre la que se estructuran organizaciones y prácticas de acción política específicas. Como una respuesta a estas condiciones, pero también por cálculos estratégicos, los actores políticos y en particular los partidos tendieron a recluirse en espacios regionales o provinciales, hasta convertirlos en verdaderos bastiones electorales (en el sentido de fortalezas a las que no pueden penetrar los otros, pero también de las que resulta difícil salir). Por ello, los partidos tradicionales que predominaron a lo largo de casi tres décadas fueron perdiendo el carácter nacional para convertirse en organizaciones que obtenían su votación mayoritariamente en una sola provincia o en un conjunto muy restringido de éstas. ${ }^{29}$ La crisis en que se sumieron desde el año 2002 se explica en gran medida por esta pérdida de su condición de organizaciones asentadas en una votación distribuida en todo el territorio nacional.

Las elecciones del año 2007 para la Asamblea Constituyente marcaron el punto de quiebre en este aspecto cuando Alianza País se convirtió en partido hegemónico con una votación proporcionalmente distribuida en términos territoriales (López, 2009). Las elecciones del año 2009 mantuvieron esa tendencia en lo que hace relación a la votación de AP y en menor medida del PSP, pero no se puede sostener lo mismo del resto de agrupaciones políticas, ya que cada una de ellas se concentró en un número reducido de provincias. Por consiguiente, se configuraron nuevamente las condiciones para que la representación legislativa sea vista como un espacio para el procesamiento de las demandas provinciales y cantonales. De esta manera, reaparecieron las viejas prácticas que impulsan a que el Legislativo se convierta en un instrumento de presión sobre el Ejecutivo.

Sin embargo, en esta ocasión, a diferencia de las anteriores, están presentes dos elementos que tienen efectos en la relación entre el Ejecutivo y las instancias subnacionales. En primer lugar, a pesar de la pérdida de la mayoría en la Asamblea, el gobierno cuenta con los recursos suficientes para obtener el control de la negociación. La debilidad de los partidos y agrupaciones de oposición sumada a la centralidad de la figura presidencial y a la reducción de las atribuciones de la Asamblea, han permitido que se establezca una relación directa entre el presidente de la República y los organismos locales y provinciales. En este aspecto es determinante el modelo centralista que impulsa el gobierno, ya que coloca a los consejos provinciales y a los municipios en una situación de supeditación, en la que prácticamente han perdido la capacidad de interlocución que tenían anteriormente. ${ }^{30}$ En

29 A manera de ejemplo, en el año 2002 el Partido Social Cristiano obtuvo el primer lugar en las elecciones legislativas -tanto en votos como en escaños- pero el 78,5\% de su total nacional lo consiguió en la provincia de Guayas (que contiene al 28\% del electorado) (Pachano, 2006)

30 Una expresión de las prácticas gubernamentales es la realización de los gabinetes itinerantes, que consisten en reuniones del gabinete presidencial realizadas una vez al mes en diversas localidades del país. En estos, el Presidente, sus ministros, secretarios y más funcionarios reciben a representantes de organizaciones previamente seleccionadas por el equipo gubernamental, escuchan sus planteamientos y definen las acciones inmediatas. Los municipios y consejos provinciales encabezados por miembros de AP son invitados a participar, no así los que están en manos de otros partidos. 
segundo lugar, la supeditación del Legislativo al Ejecutivo le ha disminuido la capacidad que el primero tenía anteriormente para convertirse en la arena política en que se procesaban los conflictos derivados de las demandas subnacionales.

\section{EVALUACIÓN GENERAL SOBRE EL FUNCIONAMIENTO Y CALIDAD DE LA DEMOCRACIA}

La vigencia de una nueva Constitución y la elección y tomada posesión de autoridades al amparo de ella podrían inducir a pensar en el inicio de una etapa radicalmente diferente a las que la antecedieron. Más aún, si se califica a esos hechos como los hitos fundamentales -y fundacionales- de una revolución, se puede suponer que de ellos surgirán no solamente nuevas instituciones, sino que se desprenderían también prácticas diferentes a las que prevalecieron anteriormente y que llevaron a la crisis del sistema político. En definitiva, se esperaba que todo el esfuerzo desplegado a lo largo de más de dos años condujera a la instauración de un nuevo régimen político. ${ }^{31}$ Sin embargo, los hechos sucedidos durante los primeros meses de vigencia de la Constitución y del nuevo gobierno tuvieron más similitudes que diferencias con los que caracterizaron a la época que se intenta superar. Esto se hace evidente cuando se toma como referencia -o como parámetros de análisisa los problemas que enfrentó el viejo sistema político.

En primer lugar, cabe señalar la baja capacidad que mostró el sistema político ecuatoriano para procesar los conflictos, lo que se tradujo en recurrentes crisis de gobernabilidad. La causa fundamental de ésta se encontraba en el diseño institucional que a) generaba problemas en la relación entre los poderes del Estado, b) incentivaba la fragmentación de la representación, c) daba amplio espacio para la acción de jugadores con poder de veto, d) alentaba las prácticas clientelares y corporativas y e) establecía plazos extremadamente cortos para la política (Mejía, 2002; 2009; Echeverría, 1997; Pachano, 2007; Basabe, Pachano y Mejía, 2010; Freidenberg, 2010; Sánchez, 2009). La Asamblea Constituyente, que era la oportunidad para resolver esos problemas, dejó intocadas varias causas de gran peso como el sistema electoral y los desequilibrios entre los poderes el Estado. El amplio triunfo de $\mathrm{AP}$, con la consecuente obtención de la mayoría absoluta, hizo que los asambleístas confiaran más en los factores políticos inmediatos que en los aspectos institucionales de mayor alcance. Por ello cobró valor la calificación de la Constitución -y en general del nuevo orden político-como un traje a la medida del presidente Correa, en el sentido de que la institucionalidad establecida difícilmente sería útil para condiciones políticas diferentes a las que existían en ese momento.

Los primeros meses de vigencia de este nuevo orden demuestran que esa apreciación tenía validez. Los problemas mencionados han vuelto a aparecer y amenazan con producir los efectos que llevaron a la crisis recurrente de los quince años anteriores. Ante la ausencia de dispositivos institucionales, la única respuesta posible, y eficiente hasta el momento,

31 Régimen político está entendido aquí como una dimensión más amplia que la de sistema, que la incluye a éste, pero que abarca además a los elementos propios del nivel normativo como a los que constituyen a la comunidad política. 
ha sido el liderazgo presidencial, asentado en un apoyo desconocido en la historia reciente -y posiblemente también en la historia larga- del país. Se ha configurado así un tipo de solución que parece nutrirse del decisionismo de Carl Schmitt y no de una concepción que otorgue importancia a la construcción de una nueva institucionalidad para profundizar la democracia o, incluso -en términos más pragmáticos para sus impulsores-, para asegurar la continuidad de la revolución ciudadana. De ello se deriva una gran incertidumbre acerca de las posibilidades de permanencia de este proceso más allá del período de gobierno de Rafael Correa.

En segundo lugar, el sistema político ecuatoriano enfrentó una crisis recurrente de representación, entendida como la pérdida de la dimensión nacional en la política y no como la exclusión de determinados sectores sociales. ${ }^{32}$ La debilidad de los partidos políticos, los constantes bloqueos a la toma de decisiones y el corporativismo fueron las expresiones que debían llevar necesariamente a otras definiciones institucionales y procedimentales. Sin embargo, también en este aspecto se dejó intocadas las causas y se prefirió acudir al planteamiento retórico de sustitución de la democracia representativa por una democracia participativa. Se trataba de una solución irrealizable no solamente por las dificultades prácticas que plantea esta última en una sociedad compleja, sino por la visión excluyente con que está construida la propuesta. ${ }^{33}$

Esto derivó en dos problemas. Por un lado, se debilitó a la democracia representativa al quitarles atribuciones a los órganos de representación popular, como es el caso de la Asamblea Nacional, pero también de los organismos subnacionales que deberán actuar conjuntamente con unos consejos conformados por personas que no provienen de la elección popular pero que tienen las mismas facultades de quienes han sido elegidos. Por otro, se establecieron las bases para la estatización de la participación al transformar a ésta no solamente en una función del Estado, sino también al cerrar los canales para el intercambio entre la sociedad y las instancias políticas. ${ }^{34}$ Por consiguiente, se introdujeron limitaciones a la democracia representativa, sin el correlato de la ampliación y la profundización de la democracia participativa.

La acción del gobierno se ha movido en ese campo, en el que no se han colocado los cimientos para solucionar los problemas de baja capacidad de procesamiento de las demandas y de representación. Como se ha señalado reiteradamente, la fuerza del proceso ha radicado en el liderazgo presidencial -apoyado en un intenso manejo de la comunicación política (De la Torre, 2009; De la Torre y Conaghan, 2008)- con todos los riesgos que ello supone. La

32 La capacidad de inclusión del sistema político ecuatoriano se demuestra tanto en resultados positivos, como la incorporación de los pueblos indígenas en la esfera política y la constante renovación de la elites políticas, pero también en resultados negativos, como la fragmentación de la representación y la sobrecarga de las demandas subnacionales en la arena política nacional (Pachano, 2007).

33 El carácter excluyente con que se entiende a las dos formas de democracia se expresa claramente en la instauración del Consejo de Participación y Control Social, donde se elimina la representación -y por tanto no existe la expresión de la voluntad ciudadana- y se establece una supuesta participación mediada por un concurso de merecimientos.

34 La reacción del gobierno ante las movilizaciones indígenas y de los maestros, reseñadas antes, es una expresión de esta visión de la participación estatizada. Lo que ocurre fuera de los ámbitos señalados para la participación pasa a ser considerado subversivo y desestabilizador. 
debilidad institucional no ha encontrado correctivos e incluso Alianza País, la organización política que debía convertirse en el factor fundamental de impulso del proceso, no ha podido constituirse como un partido o por lo menos como una agrupación dotada de una estructura orgánica y con formas de democracia interna. ${ }^{35}$

En conclusión, los primeros pasos dentro del nuevo régimen político han estado cargados de incertidumbre y no auguran un futuro de estabilidad y de profundización de la democracia. La indiferencia frente a los problemas propios de la democracia representativa y la confianza depositada exclusivamente en el liderazgo personal amenazan con convertir a la actual experiencia en el período pasajero de un gobierno y no en el cambio de régimen que se anunció. Es probable que la explicación de fondo para esto se encuentre en la calificación de revolución, esto es, como un proceso que parte desde un punto inicial en el que todo el pasado resulta oprobioso y debe ser borrado. A la vez, la calificación del proceso como una revolución establece una separación tajante entre revolucionarios y contrarrevolucionarios, entre amigos y enemigos, entre la patria y la antipatria. ${ }^{36}$ Las posibilidades de acuerdos y consensos, pero también de disenso y pluralismo, son prácticamente nulas, como se desprende de la ausencia de debate político. En esas condiciones, resultan mínimas las posibilidades de profundización de la democracia.

\section{REFERENCIAS}

Basabe, Santiago. 2009. “Ecuador: reforma constitucional, nuevos actores políticos y viejas prácticas partidistas". Revista de Ciencia Política 29 (2): 381-406.

Basabe, Santiago, Simón Pachano y Andrés Mejía. 2010. “La democracia inconclusa: derechos fundamentales, instituciones políticas y rendimientos gubernamentales en Ecuador (1979-2008)". Revista de Ciencia Política 30 (1): 65-85.

De la Torre, Carlos. 2009. "Populismo radical y democracia en los Andes". Journal of Democracy en español 1 (1) 24-37.

Echeverría, Julio. 1997. La democracia bloqueada. Quito: Letras.

Freidenberg, Flavia. 2010 (en prensa). "Elecciones, partidos y comportamiento electoral en treinta años de democracia en Ecuador (1978-2009)". In Democracia en América Latina. Balance de tres décadas, edited by S. Pachano. Quito: Flacso.

Freidenberg, Flavia y Manuel Alcántara. 2001. "Cuestión regional y política en Ecuador: partidos de vocación nacional y apoyo regional". América Latina Hoy (27): 123-152.

López, Adrián. 2009. "Elecciones 2009: cómo se votó el 26 de abril". En prensa.

Oleas, Sebastián. 2009. "Una mirada al gasto social reciente", en Carta Económica, año 15, número 12, diciembre. CORDES, Quito.

Mejía, Andrés. 2002. Gobernabilidad democrática. Quito: Fundación Konrad Adenauer. sería de carácter constitutivo), sin que llegara a concretarse. La operación de sus instancias internas está sometida a los vaivenes de la coyuntura política, incluido el buró político, su órgano máximo, cuyos integrantes son cambiantes (Diario El Universo, 13 de enero de 2010: Diario El Universo, 20 de abril de 2010).

36 Es sintomática la utilización de "Patria", una canción militar creada en la secuela de la derrota frente a Perú en 1941, como distintivo de la revolución ciudadana. Por otra parte, el presidente Correa ha logrado reinsertar el término pelucones, de cuño colonial y que había caído en desuso, para referirse no solamente a los sectores de más altos ingresos o a las oligarquías, sino también a sus opositores. 
Mejía, Andrés. 2009. Informal Coalitions an Policymaking in Latin America. Ecuador in Comparative Perspective. New York: Routledge.

Pachano, Simón. 1985. “Movimientos sociales regionales". In Movimientos sociales en el Ecuador, editado por L. Verdesoto. Quito: ILDIS.

Pachano, Simón. 2007. La trama de Penélope. Quito: Flacso-Ágora Democrática-Idea-Inmd.

Pachano, Simón. 2008. "Sistemas subnacionales de partidos en el Ecuador". In Descentralizar: un derrotero a seguir, editado por F. Carrión and B. Villaronga. Quito: Flacso-Inwent-Senplades.

Quantum. "Índices de confianza ciudadana Quantum-Habitus", medio magnético. Quito, Ecuador, 20022010. Reproducidos con autorización de Serviquanti y Habitus-Estudios de Mercados y Culturas.

Sánchez, Francisco. 2008. ¿Democracia no lograda o democracia malograda? Un análisis del sistema político del Ecuador: 1979-2002. Quito, Flacso.

Shugart, Matthew, and John Carey. 1992. Presidents and Assemblies: Constitutional Design and Electoral Dynamics. Cambridge: Cambridge University Press.

Simón Pachano. Doctor en Ciencia Política por la Universidad de Salamanca. Coordinador del Doctorado en Estudios Andinos, Flacso, Ecuador. 
